\title{
Time Quantum based CPU Scheduling Algorithm
}

\author{
Mayank Parashar \\ M.Tech Scholar \\ Department of CSE, MRIU
}

\author{
Amit Chugh \\ AssociateProfessor \\ Department of CSE, MRIU
}

\begin{abstract}
The objective of this paper is to introduce a new CPU Scheduling Algorithm called time quantum based CPU Scheduling Algorithm which acts as preemptive based on the arrival time. The algorithm helps to improve the average waiting time of Round Robin algorithm in real time uniprocessor-multi programming operating system. CPU Scheduling is the basis of multi-programmed operating system. The scheduler is responsible for multiplexing processes on the CPU. There are many scheduling algorithms available for a multi-programmed operating system like FCFS, SJF, Priority, Round Robin etc. The proposed algorithm is based on Round robin scheduling . In this paper, the results of the existing Round Robin algorithm is compared with the proposed algorithm
\end{abstract}

\section{General Terms}

CPU Scheduling Algorithms, Context Switching.

\section{Key Words}

Round Robin Scheduling, Waiting Time, Turn Around time, Average waiting Time, Average Turn Around time, Context Switches.

\section{INTRODUCTION}

Operating system performs variety of tasks in which scheduling is one of the basic task. Scheduling is heart of any computer system since it contains decision of giving resources between possible processes. Sharing of computer resources between multiple processes is also called scheduling. Process is a smallest work unit of a program which requires a set of resources for its execution that are allocated to it by the CPU. These processes are many in number and keep coming in a particular fashion, different scheduling techniques are employed that enable faster and efficient process execution thereby reducing the waiting time faced by each process and increasing CPU utilization.

\section{SCHEDULING PARAMETERS}

There are different scheduling algorithm with different characteristics which decide selection of process using different criteria for execution by CPU. The Criteria for a good scheduling algorithm depends, among others, on the following measures:-

2.1. CPU Utilization: It is the average fraction of time, during which the processor is busy .

2.2. Throughput: It refers to the amount of work completed in a unit of time. The number of processes the system can execute in a period of time. The higher the number, the more work is done by the system .

2.3. Waiting Time: The average period of time a process spends waiting. Waiting time may be expressed as turnaround time less the actual execution time.
2.4. Turnaround time: The interval from the time of submission of a process to the time of completion is the turnaround time.

2.5 Response time: Response time is the time from submission of a request until the first response is produced .

2.6. Priority: It give preferential treatment to processes with higher priorities .

2.7. Fairness: Avoid the process from starvation. All the processes must be given equal opportunity to execute.

\section{EXISTING CPU SCHEDULING ALGORITHMS OVERVIEW}

\subsection{Round Robin Scheduling Algorithm}

It is a preemptive scheduling algorithm. It is designed especially for time sharing systems. In this algorithm, a small unit of time called time quantum or time slice is assigned to each process. When the time quantum expired, the CPU is switched to another process. Performance of Round Robin totally depends on the size of the time quantum.

For example, Suppose we have 5 different processes with their following properties:

Table 1

\begin{tabular}{|c|c|}
\hline & \\
PROCESSES & BURST TIME \\
\hline P1 & 12 \\
\hline P2 & 15 \\
\hline P3 & 23 \\
\hline P4 & 37 \\
\hline P5 & 21 \\
\hline
\end{tabular}

Suppose for above condition let the time quantum of $10 \mathrm{~ns}$ then the Gantt Chart for Round robin scheduling is:-

\begin{tabular}{|l|l|l|ll|ll|l|l|}
\hline $\mathrm{P} 1$ & $\mathrm{P} 2$ & $\mathrm{P} 3$ & $\mathrm{P} 4$ & $\mathrm{P} 5$ & $\mathrm{P} 1$ & $\mathrm{P} 2$ \\
\hline 0 & 10 & 20 & 30 & 40 & 50 & 52 & 57
\end{tabular}




\begin{tabular}{|c|c|c|c|c|c|c|}
\hline P3 & P4 & P5 & P3 & P4 & P5 & P4 \\
\hline 67 & 77 & 87 & 90 & 100 & 101 & 108
\end{tabular}

Waiting time

$=[52-12]+[57-15]+[90-23]+[108-37]+[101-21]$

$=40+42+67+71+80$

$=300$

Average Waiting time $=300 / 5=60$

Turn around time

$=52+57+90+108+101$

$=408$

Average turn around time $=408 / 5=81.6$

\subsection{Shortest Job Round Robin Algorithm}

Select the sequence of process according to the to burst time. Means the process having minimum burst time should be select first then 2 nd one .The process which has minimum burst time should be used as time quantum. When we arrange the processes into increasing order to their burst time the average waiting time is going improve which is shown in bellow. The quanta should be equal to the shortest process which may affect to response time but due to this we can improve the average waiting time which is more important.

Refer to Table 1

Table 2

\begin{tabular}{|c|c|}
\hline & \\
\hline & \\
PROCESSES & BURST TIME \\
\hline & \\
\hline P1 & 12 \\
\hline P2 & 15 \\
\hline & \\
P5 & 21 \\
\hline & \\
\hline P3 & 23 \\
\hline & \\
\hline P4 & 37 \\
\hline
\end{tabular}

In this example, $\mathrm{P} 1$ process has minimum burst time, we use minimum burst time as a time quantum. The gantt chart is:-

\begin{tabular}{|l|l|l|ll|ll|l|}
\hline P1 & P2 & P5 & P3 & P4 & P2 & \\
\hline 0 & 12 & 24 & 36 & 48 & 60 & 63
\end{tabular}

\begin{tabular}{|c|c|c|l|c|}
\hline P5 & P3 & P4 & P4 & P4 \\
\hline 72 & 83 & 95 & 107 & 108
\end{tabular}

Waiting time

$=0+[63-15]+[72-21]+[83-23]+[108-37]$

$=0+48+51+60+71=230$

Average Waiting Time $=230 / 5=46$

Turn Around Time $=12+63+108+83+72=338$
Average Turn Around Time=338/5 =67.6

\subsection{Enhance Round Robin Algorithm}

In this approach ,it does not aim to change the philosophy of the conventional round robin algorithm but to enhance it further. In our proposed approach, we will be modifying the time quantum of only those processes which require a slightly greater time than the allotted time quantum cycle(s). The remaining processes will be executed in the conventional Round Robin manner.

Refer to Table 1(a) the Gantt chart for the Example is:-

\begin{tabular}{|c|c|c|c|c|c|}
\hline P1 & P2 & P3 & $\mathrm{P} 4$ & P5 & $\mathrm{P}$ \\
\hline 0 & 12 & 22 & 32 & 42 & 52 \\
\hline P3 & P4 & & P5 & P3 & P4 \\
\hline
\end{tabular}

Waiting time

$=[12-12]+[57-15]+[91-23]+[108-37]+[88-21]$

$=248$

Average waiting time $=248 / 5=49.6$

Turn around time

$=12+57+91+108+88$

$=356$

Average turn around time $=356 / 5=71.2$

3.4Adaptive Round Robin Algorithm:

In this algorithm,if the number of processes are odd then we select the mid one process burst time and use this process burst time as a time quantum and if the no of processes are even then we calculate the time quantum from average mean.

Refer to Table 1(a) the Gantt chart for the Example is:-

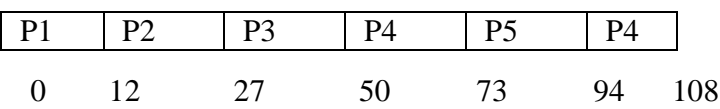

Waiting time

$=0+[27-15]+[50-23]+[108-37]+[94-21]$

$=12+27+71+73=183$

Average Waiting Time $=183 / 5=36.6$

Turn Around time

$=12+27+50+108+94=291$

Average Turn Around Time $=291 / 5=58.2$

\section{PROPROSEDWORK: TIME QUANTUM BASED CPU SCHEDULING ALGORITHM}

The proposed Time quantum based CPU Scheduling algorithm is preemptive in nature and based on Round robin scheduling mechanism. In this algorithm we will try to improve the waiting time, average waiting time, turn around time, average turn around time of Round robin scheduling by applying certain rules and regulation. In this research we try to show the comparison between traditional Round robin scheduling technique to proposed algorithm. 
4.1The pseudo code of proposed algorithm is

1. Assume $p_{i}$ and $b_{i}$ are the processes and their respective burst time where $i=(1$ to.... $n)$.

2. Arrange the processes in increasing order of burst time such that $\left(b_{i+1} \geq b_{i}\right)$ where $i$ is the no of process and value of $i=(1$ to $\mathrm{n})$.

3. Calculate n-1

$$
\sum_{i=1}\left(b_{i+1}-b_{i}\right)
$$

4. After calculating the value from step 3.Use this value as a time quantum and perform calculation as usually by round robin scheduling and obtain better results.

\subsection{Steps}

1. Arrange the sequence of the processes according to their burst time. Mean the process which have minimum burst time should be selected first then second one. When we arrange the process in increasing order according to their burst time the average waiting time is going to improve which is shown below:-

2. Then subtract the burst time of the first process from the second one, second process burst time from the third and so on until we receive the no of new values less one than the no .of process.

3.After receiving the values less than the no of processes. Adding these values and use the new added value as a time quantum and then we will receive the better results as compared to the classical round robin scheduling algorithm.

Example

Refer to table 1(a).The Modified Table is

Table 3

\begin{tabular}{|c|c|}
\hline PROCESSES & BURST TIME \\
\hline P1 & 12 \\
\hline P2 & 15 \\
\hline P5 & 21 \\
\hline P3 & 23 \\
\hline P4 & 37 \\
\hline
\end{tabular}

2. Subtract the processes values from the next one.

$=[15-12]+[21-15]+[23-21]+[37-23]$

$=3+6+2+14=25$

3. Time quantum $=25$

Then Gantt chart is

\begin{tabular}{|l|l|l|l|l|ll|}
\hline 0 & 12 & 27 & 48 & 71 & 96 & 108 \\
\hline P1 & P2 & P5 & P3 & P4 & P4 \\
\hline
\end{tabular}

Waiting Time

$=[0]+[27-15]+[71-23]+[108-37]+[48-21]$

$=0+12+48+71+27$

$=158$

Average Waiting time $=158 / 5=31.6$

Turn around time

$=12+27+71+108+48$

$=266$

Average Turn Around Time $=266 / 5=53.2$

\section{COMPARISON BETWEEN ROUND ROBIN,OTHER VARIENTS OF ROUND ROBIN AND TIME QUANTUM BASED CPU SCHEDULING ALGORITHM}

\begin{tabular}{|l|c|c|c|c|}
\hline \multicolumn{1}{|c|}{ Algorithm } & WT & AWT & TA & ATA \\
\hline $\begin{array}{l}\text { Round Robin } \\
\text { Scheduling } \\
\text { Algorithm }\end{array}$ & 300 & 60 & 408 & 81.6 \\
\hline $\begin{array}{l}\text { Shortest Job } \\
\text { Round Robin } \\
\text { Algorithm }\end{array}$ & 230 & 46 & 338 & 67.6 \\
\hline $\begin{array}{l}\text { Enhanced } \\
\text { Round Robin } \\
\text { Algorithm }\end{array}$ & 248 & 49.6 & 356 & 71.2 \\
\hline $\begin{array}{l}\text { Adaptive } \\
\text { Round Robin } \\
\text { Scheduling } \\
\text { Algorithm }\end{array}$ & 183 & 36.6 & 291 & 58.2 \\
\hline $\begin{array}{l}\text { Time } \\
\text { Quantum } \\
\text { Based } \\
\text { CPU } \\
\text { Scheduling } \\
\text { Algorithm }\end{array}$ & $\mathbf{1 5 8}$ & $\mathbf{3 1 . 6}$ & $\mathbf{2 6 6}$ & $\mathbf{5 3 . 2}$ \\
\hline
\end{tabular}




\section{GRAPHICAL COMPARISON B/W DIFFERENT ALGORITHMS}

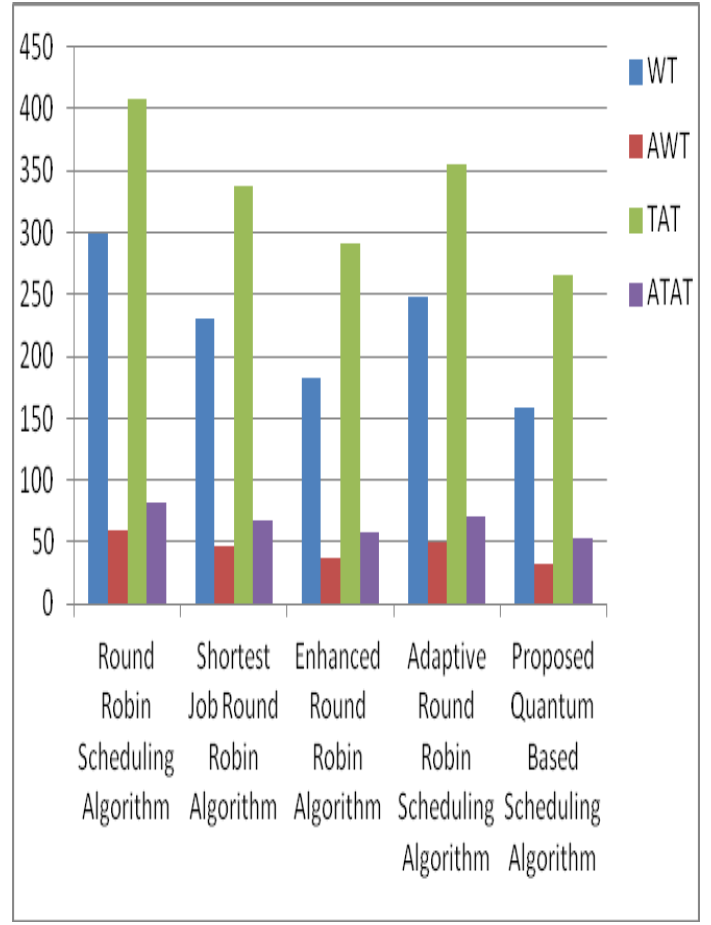

\section{ACKNOWLEDGMENTS}

Our thanks to the Professors, experts and other faculty members who provided useful resources and background to complete this research paper

\section{CONCLUSION}

The paper presents a new CPU scheduling algorithm called Time Quantum Based CPU Scheduling Algorithm which is based on traditional Round Robin algorithm .Paper also contains the comparison of proposed algorithm with traditional Round Robin algorithm. The result of the comparison for different process sets using different scheduling algorithms. The Paper also explain how proposed algorithm improve the performance of traditional one. The proposed variant of $\mathrm{RR}$ algorithm drastically decreases context switching. The proposed algorithm performs better than RR scheduling algorithm with respect to average waiting time, turnaround time and context switching. Our proposed algorithm can be further investigated to be useful in providing more and more task-oriented results in future along with developing adaptive algorithms to fit the varying situations in today's multifaceted complex working of operating system.

\section{REFERENCES}

[1].Rakesh Patel,Mrs Milli Patel Shortest Job Round Robin $\mathrm{Cpu}$ Scheduling Algorithm International Journal of
Engineering and Computer Science Volume 2 December 2013.

[2].Aashna Bisht ,MD.Abdul Azad, SielVie Sharma, Enhanced Round Robin for Process scheduling using varying Quantum Decision IRAJ International Conference Proceedings December 2013.

[3].Abbas Noon,Ali KalaKech, Seifedine Kadry A New Round Robin Based Scheduling Algorithm for Operating Systems: Dynamic Quantum Using the Mean Average International Journal of computer science Volume 8 May 2013.

[4].Hemant M.Chaskar Upamanyu Madhow, Senior Member, IEEE A Fair scheduling with tuneable Latency:A Rond Robin Approach IEEE Transcations August 2003

[5]. P. SurendraVerma Improved Shortest Remaining Burst Round Robin (ISRBRR) Using RMS as its time Quantum International Journal of Advanced Research in Computer Engineering \& Technology (IJARCET)Volume 1, Issue 8, October 2012.

[6]. SAROJ HIRANWAL "Adaptive Round Robin Scheduling using Shortest Burst ApproachBased on Smart Time Slice" International Journal of Data Engineering (IJDE), Volume 2, Issue 3 MaY 2013.

[7]Krithi Ramamaritham John A Stankovic Senior Member IEEE And Perg Fersiah Efficient Scheduling Algorithms for Real time Multiprocessor Systems Transctions on Parallel and Distributed systems Vol.2 No 1 ,April 1990

[8]Bashir Alam, Fuzzy Round Robin Cpu Scheduling Algorithm, Journal of Computer Science, 9(8):10791085,2013 Science Publications.

[9]Superiya Raheja, Reena Dhadich, Smita Rajpal, An Optimum Time Quantum Using Linguistic Synthesis for Round Robin Cpu Scheduling Algorithm, International Journal on Soft Computing(IJSC) Vol.3 No.1 Feburary 2012.

[10]C.Yashuwanth and R.Ramesh,: A new Scheduling Algorithm For Real Time System , International Journal of Computer and Electrical Engineering(IJCEE)Vol 2 No.6,pp 1104-1106, December,2010.

[11] Abraham Silberschatz, Peter Baer Galvin, Greg Gagne, "Operating System Concepts", Sixth Edition.

[12] Milan Milenkovic, "Operating Systems Concepts and Design", McGRAM-HILL, Computer Science Series, second edition.

[13] P. Balakrishna Prasad, "Operating Systems" Second Edition.

[14] A. Dhore "Opeating Systems", Technical Publications.

[15] M. Dietel, "Operating Systems", Pearson Education, Second Edition.

[16] M Gary Nutt, "Operating systems - A Modern Perspective, Second Edition, Pearson Education, 2000. 